Polymer Journal, Vol. 2, No. 3, pp 416-421 (1971)

\title{
Excluded-Volume Effect on Dipole Moments of Polymer Chains
}

\author{
Kazuo NAGaI and Toshihide Ishikawa \\ Government Industrial Research Institute, Osaka, \\ Midorigaoka 1, Ikeda, Osaka, Japan. \\ (Received February 12, 1971)
}

\begin{abstract}
The excluded-volume effect on dipole moments of linear polymer chains is investigated by the perturbation method. Fixman's formulation is followed here except for molecular models. In place of the multi-Gaussian-subchain model a more realistic model is adopted. The expansion factor $\alpha_{u}$ for the dipole moment is found, up to the term in $z^{3}$, to be

$$
\alpha u^{2}=1+\left(C_{1} z+C_{2} z^{2}+C_{3} z^{3}+\cdots\right)\left(\langle\mathbf{r} \cdot \mathbf{u}\rangle_{0}^{2}\left\langle\left\langle\boldsymbol{r}^{2}\right\rangle_{0}\left\langle u^{2}\right\rangle_{0}\right)\right.
$$

where $\mathbf{r}$ and $\mathbf{u}$ are the end-to-end and dipole-moment vectors, $\alpha u^{2}=\left\langle u^{2}\right\rangle\left\langle u^{2}\right\rangle_{0}$, the averages without and with the subscript 0 refer to those in the presence and in the absence of the excluded volume, and $C$ 's and $z$ are the familiar quantities in the similar expression for the end-to-end distance, $\alpha r^{2}=1+C_{1} z+C_{2} z^{2}+C_{3} z^{3}+\cdots$. This result strongly suggests

$$
\alpha u^{2}-1=\left(\langle\mathbf{r} \cdot \mathbf{u}\rangle_{0}^{2} /\left\langle r^{2}\right\rangle_{0}\left\langle u^{2}\right\rangle_{0}\right)\left(\alpha_{r}{ }^{2}-1\right)
$$
\end{abstract}

An expression for $\langle\mathbf{r} \cdot \mathbf{u}\rangle_{0}$ is given on the basis of the rotational-isomeric-state approximation for skeletal-bond rotations. From a structural symmetry consideration it is concluded that $\langle\mathbf{r} \cdot \mathbf{u}\rangle_{0}=0$ and hence the excluded volume has no effect on dipole moments for polymers with no distinguishable direction along chain contours.

KEY WORDS Excluded Volume / Dipole Moment / Polymer Chain / Perturbation Theory/Chain Dimension / Realistic Model / Structural Symmetry /

The exclude-volume effect on chain dimensions of polymer chains in dilute solution has been extensively investigated from both experimental and theoretical points of view, and is fairly well understood at present. ${ }^{1,2}$ In contrast, the effect on dipole moments has been much less studied and hence only poorly understood. It has been known experimentally ${ }^{3-7}$ that the excluded volume has little effect on dipole moments, in sharp contrast with its pronounced effect on chain dimensions. Marchal and Benoit ${ }^{6}$ showed, based on a very crude model, that the effect on dipole moments should vanish when a resultant dipole-moment vector of a repeating unit is directed along the bisector of the bond angle of the chain skeleton. No rigorous theoretical reasoning for their conjecture has yet been reported.

We have investigated the effect by the perturbation method. This method has been well de- veloped by Teramoto ${ }^{8}$ and many others in connection with chain dimensions. Fixman's formulation $^{9}$ has been followed except for the molecular models. In place of the multi-Gaussiansubchain model used by him, ${ }^{9}$ a more general model, which comprehends all hypothetical and realistic models, is adopted. (A perturbation treatment was developed by $\mathrm{Oku}^{10}$ and then extended by Okano and Tanabe, ${ }^{11}$ based on the multi-Gaussian-subchain model, i.e., with neglect of realistic structures of polymer chains. For their model, the consequences of our and their respective theories are not inconsistent. Our theory predicts $C_{r u}=0$ and hence $\alpha_{u}=1$. Terms in $\beta$ and $\beta^{2}$, retained by them, ${ }^{10,11}$ are of secondary importance, vanishing as $N$ tends to infinity while $z$ remains finite.) We have obtained a very interesting relation of the excluded-volume effect on dipole moments with that on chain dimensions. We report these results and some 
additional consequences of them in this paper.

\section{PERTURBATION THEORY}

We consider a linear flexible polymer chain composed of $N$ repeating units (or properly chosen segments) numbered $1,2, \ldots, N$ from one end to the other. Any more precise structure of the polymer chain need not be defined at this stage. Replacing the end-to-end vector $\mathbf{r}$ with the dipolemoment vector $\mathbf{u}$ in Fixman's formulation ${ }^{9}$ we find for the mean-square dipole moment

$$
\begin{aligned}
\left\langle u^{2}\right\rangle= & \left\langle u^{2}\right\rangle_{0}-\beta \sum_{i<j} \int u^{2}\left[P\left(\mathbf{u}, 0_{i j}\right)-P(\mathbf{u}) P\left(0_{i j}\right)\right] \mathrm{d} \mathbf{u} \\
& +\beta^{2} \sum_{\substack{i<j \\
i<l k}} \sum_{\substack{k<l \\
i}} \int u^{2}\left[P\left(\mathbf{u}, 0_{i j}, 0_{k l}\right)-P(\mathbf{u}) P\left(0_{i j}, 0_{k l}\right)\right. \\
& +2 P(\mathbf{u}) P\left(0_{i j}\right) P\left(0_{k l}\right)-P\left(\mathbf{u}, 0_{i j}\right) P\left(0_{k l}\right) \\
& \left.-P\left(\mathbf{u}, 0_{k l}\right) P\left(0_{i j}\right)\right] \mathrm{d} \mathbf{u}+\cdots
\end{aligned}
$$

where the averages with and without the subscript 0 refer to those in the absence and in the presence of the excluded volume; $\beta$ is the binary cluster integral for units (or segments); $\boldsymbol{P}(\mathbf{w}), P\left(\mathbf{u}, \mathbf{r}_{i j}\right)$, $\boldsymbol{P}\left(\mathbf{u}, \mathbf{r}_{i j}, \mathbf{r}_{k l}\right)$ are the unperturbed, monovariate, bivariate, trivariate distribution functions, $\mathbf{r}_{i j}$ being the vector from unit $i$ to unit $j$; and $\boldsymbol{P}\left(\mathbf{u}, 0_{i j}\right)=\boldsymbol{P}\left(\mathbf{u}, \mathbf{r}_{i j}\right)$ at $\mathbf{r}_{i j}=0$, etc. The term in $\beta^{3}$ in eq 1 can be obtained from the work of Yamakawa and Tanaka. ${ }^{12}$

Fixman $^{9}$ used the multi-Gaussian-subchain model, i.e., a chain composed of many Gaussian subchains. Instead we adopt a more general model which comprehends all hypothetical and realistic models. The necessary change in theory is only of nominal character, but final results become much more general thereby. We assume $N$ to be so large that we can assume u to obey a Gaussian distribution. Not only for u but also for $\mathbf{r}_{i j}$ do we assume a Gaussian distribution. This assumption is not valid for small $j-i$ for a realistic chain, but contributions from such pairs to $\left\langle u^{2}\right\rangle$ become negligible at the limit $N \rightarrow \infty$. A multivariate distribution function $P\left(\mathbf{v}_{1}, \mathbf{v}_{2}, \ldots, \mathbf{v}_{t}\right)$ of $t$ vectors for a real chain in this Gaussian approximation can be obtained by an intuitive generalization of Fixman's result (Ref 9, Appendix):

$$
\begin{aligned}
P\left(\mathbf{v}_{1}, \ldots, \mathbf{v}_{t}\right)= & (3 / 2 \pi)^{3 t / 2} q^{-3 / 2} \\
& \times \exp \left[(-3 / 2 q) \sum_{i=1}^{t} \sum_{j=1}^{t} q^{i j}\left(\mathbf{v}_{i} \cdot \mathbf{v}_{j}\right)\right]
\end{aligned}
$$

where $q$ is the determinant of $\mathbf{q}=\left\{q_{i j}\right\}=\left\{\left\langle\mathbf{v}_{i} \cdot \mathbf{v}_{j}\right\rangle_{0}\right\}$ and $q^{i j}$ is a cofactor of $q_{i j}$ in $\mathbf{q}$. $\mathbf{A}$ rigorous derivation of eq 2 is given in the Appendix. For $P(\mathbf{u})$ and $P\left(\mathbf{u}, 0_{i j}\right)$ we immediately obtain

$$
\begin{aligned}
P(\mathbf{u})= & \left(3 / 2 \pi N C_{u u}\right)^{3 / 2} \exp \left(-3 u^{2} / 2 N C_{u u}\right) \\
P\left(\mathbf{u}, 0_{i j}\right)= & (3 / 2 \pi)^{3 / 2}\left[N(j-i) C_{r r} C_{u u}-(j-i)^{2} C_{r u}^{2}\right]^{-3 / 2} \\
& \times \exp \left\{-3(j-i) C_{r r} u^{2} / 2\left[N(j-i) C_{r r} C_{u u}\right.\right. \\
& \left.\left.-(j-i)^{2} C_{r u}^{2}\right]\right\}
\end{aligned}
$$

where

$$
\begin{gathered}
C_{u u}=\lim _{N \rightarrow \infty} N^{-1}\left\langle u^{2}\right\rangle_{0}, \quad C_{r r}=\lim _{N \rightarrow \infty} N^{-1}\left\langle r^{2}\right\rangle_{0}, \\
\quad \text { and } \quad C_{r u}=\lim N^{-1}\langle\mathbf{r} \cdot \mathbf{u}\rangle_{0}
\end{gathered}
$$

In obtaining eq 4 we used $\left\langle r_{i j}^{2}\right\rangle_{0}=(j-i) C_{r r}$ and

$$
\begin{aligned}
\left\langle\mathbf{u} \cdot \mathbf{r}_{i j}\right\rangle_{0}= & \left\langle\mathbf{u}_{1 i} \cdot \mathbf{r}_{i j}+\mathbf{u}_{i j} \cdot \mathbf{r}_{i j}+\mathbf{u}_{j N} \cdot \mathbf{r}_{i j}\right\rangle_{0} \\
& =\left\langle\mathbf{u}_{i j} \cdot \mathbf{r}_{i j}\right\rangle_{0}=(j-i) C_{r u}
\end{aligned}
$$

Other higher-order distribution functions can be obtained similarly, but are inevitably more complex. We have seen that all realistic features of the chain are absorbed into $C_{u u}, C_{r r}$, and $C_{r u}$, and no other kinds of average appear.

The integrations in eq 1 are elementary with Gaussian functions for distribution functions. The terms in $\beta, \beta^{2}$, and $\beta^{3}$ are found, upon integration, to be equal to the corresponding terms in a similar expression for the end-to-end distance, except for the factor $C_{r u}^{2} / C_{r r}$ multiplied. We thus find

$$
\begin{aligned}
\alpha_{u}{ }^{2}= & \left\langle u^{2}\right\rangle\left\langle\left\langle u^{2}\right\rangle_{0}\right. \\
= & 1+\left(C_{1} z+C_{2} z^{2}+C_{3} z^{3}+\cdots\right) \\
& \times\left(\langle\mathbf{r} \cdot \mathbf{u}\rangle_{0}{ }^{2} /\left\langle r^{2}\right\rangle_{0}\left\langle u^{2}\right\rangle_{0}\right)
\end{aligned}
$$

Here $C_{r u}^{2} / C_{r r} C_{u u}$ was replaced by $\langle\mathbf{r} \cdot \mathbf{u}\rangle_{0}^{2} /$ $\left\langle r^{2}\right\rangle_{0}\left\langle u^{2}\right\rangle_{0}$. Therefore the averages in eq 6 should be taken with the unperturbed chain of infinite length. $C_{1}, C_{2}, C_{3}$, and $z$ are those in the similar expression for the end-to-end distance:

$$
\alpha_{r}^{2}=1+C_{1} z+C_{2} z^{2}+C_{3} z^{3}+\cdots
$$

where $z=\left(3 / 2 \pi C_{r r}\right)^{3 / 2} \beta N^{1 / 2}, \quad C_{1}=4 / 3$ is due to Teramoto, ${ }^{8} C_{2}=-2.075$ due to Fixman, ${ }^{9}$ and $C_{3}=$ 6.459 due to Yamakawa and Tanaka. ${ }^{12}$ Though 
eq 6 is confirmed to be valid up to the term in $z^{3}$, it is quite likely that it is valid for every term. If so, we have the interesting relation

$$
\left(\alpha_{u}{ }^{2}-1\right)=\left(\langle\mathbf{r} \cdot \mathbf{u}\rangle_{0}{ }^{2} /\left\langle r^{2}\right\rangle_{0}\left\langle u^{2}\right\rangle_{0}\right)\left(\alpha_{r}{ }^{2}-1\right)
$$

\section{CALCULATION OF $\langle\mathbf{r} \cdot \mathbf{u}\rangle_{0}^{2} /\left\langle r^{2}\right\rangle_{0}\left\langle u^{2}\right\rangle_{0}$ FOR REALISTIC MODELS}

Methods for calculating $\left\langle r^{2}\right\rangle_{0}$ and $\left\langle u^{2}\right\rangle_{0}$ for realistic models with fixed bond lengths and angles and hindered, interdependent internal rotations, have been well established, ${ }^{13-15}$ and these methods are readily adaptable to $\langle\mathbf{r} \cdot \mathbf{u}\rangle_{0}$. Hence, omitting details of derivation we record only final results. We establish the identity

$$
\langle\mathbf{r} \cdot \mathbf{u}\rangle_{0}=\frac{1}{2}\left[\langle(\mathbf{r}+\mathbf{u}) \cdot(\mathbf{r}+\mathbf{u})\rangle_{0}-\left\langle r^{2}\right\rangle_{0}-\left\langle u^{2}\right\rangle_{0}\right]
$$

We obtain for a polymer chain of $n$ skeletal bonds

$$
\begin{aligned}
& \langle(\mathbf{r}+\mathbf{u}) \cdot(\mathbf{r}+\mathbf{u})\rangle_{0}=Z^{-1}\left(\mathbf{e}_{s}{ }^{T} \quad \mathbf{0} \quad \mathbf{0}\right) \mathbf{H}_{1} \mathbf{H}_{2} \cdots \mathbf{H}_{n}\left[\begin{array}{c}
\mathbf{0} \\
\mathbf{0} \\
\mathbf{e}_{s}
\end{array}\right] \\
& \mathbf{H}_{i}=\left[\begin{array}{ccc}
\mathbf{p} & 2\left[\mathbf{E}_{s} \times(\mathbf{b}+\mathbf{m})^{T}\right] \tilde{\mathbf{A}} & (\mathbf{b}+\mathbf{m}) \cdot(\mathbf{b}+\mathbf{m}) \mathbf{p} \\
\mathbf{0} & \tilde{\mathbf{A}} & \mathbf{p} \times(\mathbf{b}+\mathbf{m}) \\
\mathbf{0} & \mathbf{0} & \mathbf{p}
\end{array}\right]_{i}
\end{aligned}
$$

where $Z=\mathbf{e}_{s}{ }^{T} \mathbf{p}_{1} \mathbf{p}_{2} \ldots \mathbf{p}_{n} \mathbf{e}_{s}$ is the partition function of the free, unperturbed chain, $\mathbf{p}_{i}$ is the statisticalweight matrix for skeletal bonds $i$ and $i+1$, and $\mathbf{b}_{i}$ and $\mathbf{m}_{i}$ are the bond vector of the skeletal bond $i$ and the dipole-moment vector of group $i$, both expressed in coordinate system $i$ fixed to the framework of skeletal bonds $i-1$ and $i$. For undefined symbols and notations see elsewhere. ${ }^{13,14}\left\langle r^{2}\right\rangle_{0}$ or $\left\langle u^{2}\right\rangle_{0}$ is obtained from eq 10 by putting $\mathbf{u}=0$ or $\mathbf{r}=0$. As pointed out, the averages $\langle\mathbf{r} \cdot \mathbf{u}\rangle_{0}$, etc., should be taken with the chain of infinite length. Asymptotic expressions at $N \rightarrow \infty$ for the averages, not in the form of the product of $n$ H's like eq 10, can be obtained analytically for stereoregular chains. ${ }^{13,14}$

\section{APPLICATION AND DISCUSSION}

Some interesting features are apparent from eq 6 or 8 . If $\langle\mathbf{r} \cdot \mathbf{u}\rangle_{0}=0$, then $\alpha_{u}=1$ irrespective of $z$. This fact was pointed out by Stockmayer without rigorous proof. ${ }^{16}$ If the excluded volume acts as $\alpha_{r}>1$, then it makes $\alpha_{u}>1$. This result is not necessarily obvious without theory. Since $0 \leq(\mathbf{r} \cdot \mathbf{u})^{2} / r^{2} u^{2} \leq 1$ for every conformation it is quite likely that

$$
0 \leq\langle\mathbf{r} \cdot \mathbf{u}\rangle_{0}^{2} /\left\langle r^{2}\right\rangle_{0}\left\langle u^{2}\right\rangle_{0} \leq 1
$$

and hence

$$
\left|\alpha_{u}{ }^{2}-1\right| \leq\left|\alpha_{r}^{2}-1\right|
$$

It is also likely that the first and second equalities in eq 12 , respectively, hold only when $\mathbf{u}$ is perpendicular to, and parallel with $\mathbf{r}$ for every conformation. We have not yet found rigorous proofs of eq 12 and 13.

As an example we have calculated $\langle\mathbf{r} \cdot \mathbf{u}\rangle_{0}$ for poly(oxyethylene), based on a model explored by Mark and Flory, ${ }^{17,18}$ and have found it to be exactly zero for some examined sets of statistical-weight parameters. This result appears to be a necessary consequence of the structural symmetry of this polymer. For polymers with some particular structural symmetries, including poly(oxyethylene), we can prove $\langle\mathbf{r} \cdot \mathbf{u}\rangle_{0}=0$ only by a symmetry consideration, as shown below.

Consider a stereoregular polymer chain composed of $N$ structural units, $N$ being assumed even. For the moment the chain is assumed to have no deformable side groups. Let this chain be in the all-trans, planar conformation, as shown in Figure 1. Suppose the chain in this conformation has either

(i) N-1 symmetry planes (Figure 1a),

(ii) $N-1$ two-fold symmetry axes (Figure 1b, chain lines), or

(iii) $N-1$ symmetry points (Figure $1 \mathrm{c}$, circles) with respect to each of which configurations and conformations of the left and right portions of the chain are symmetric except for the difference in their lengths. For this conformation $\mathbf{r} \cdot \mathbf{u}$ is zero according to the stated symmetries; $\mathbf{u}$ is perpendicular to $\mathbf{r}$ in cases (i) and (ii), and $\mathbf{u}$ is zero in case (iii).

For almost all random-coil conformations the stated symmetries are lost, but nevertheless $\langle\mathbf{r} \cdot \mathbf{u}\rangle_{0}$ should be zero because

$$
\begin{aligned}
& \left\langle\mathbf{r} \cdot \mathbf{u}_{1}\right\rangle_{0}=-\left\langle\mathbf{r} \cdot \mathbf{u}_{N}\right\rangle_{0}, \\
& \left\langle\mathbf{r} \cdot \mathbf{u}_{2}\right\rangle_{0}=-\left\langle\mathbf{r} \cdot \mathbf{u}_{N-1}\right\rangle_{0}, \ldots
\end{aligned}
$$


(a)

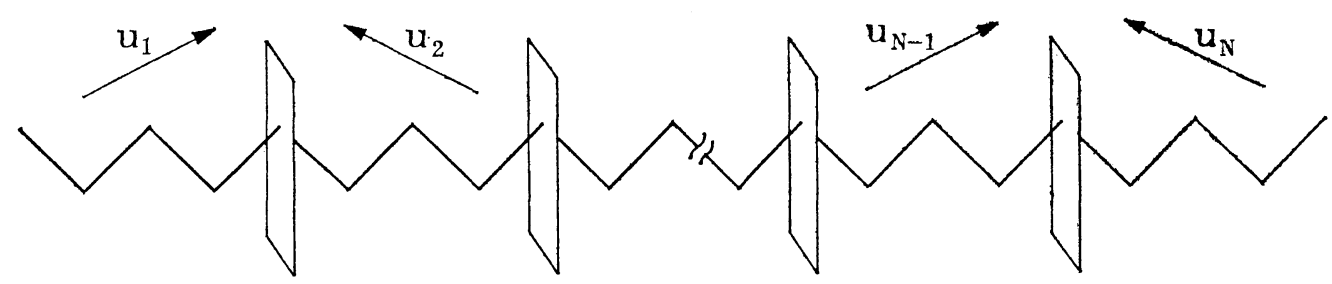

(b)

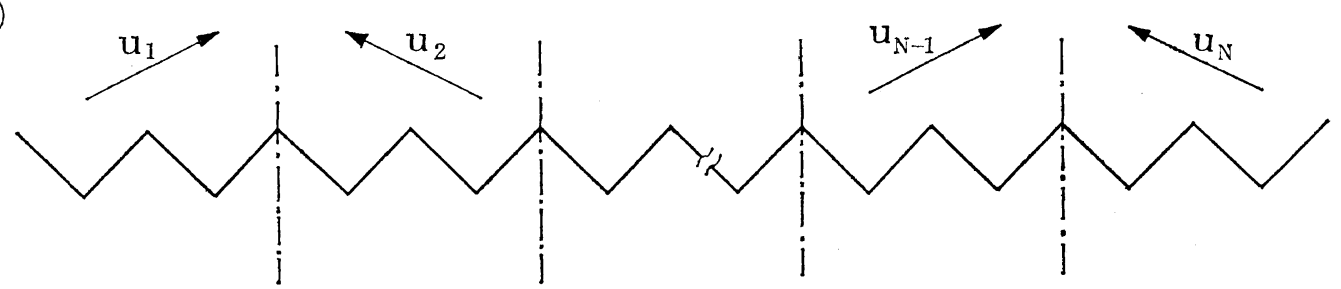

(c)

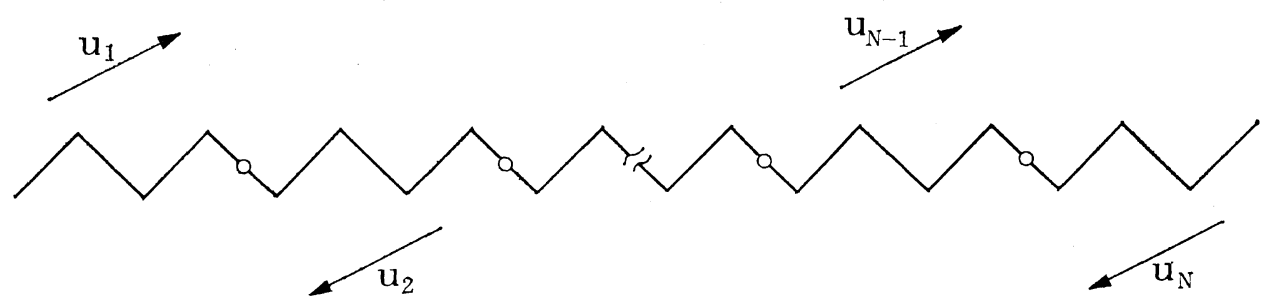

Figure 1. (a) Symmetry planes, (b) two-fold symmetry axes (chain lines), and (c) symmetry points (circles).

according to the symmetries, where $\mathbf{u}_{i}$ is the resultant dipole-moment vector of unit $i$. Isotactic poly(vinyl halide)s and polyacrylonitrile have the symmetry planes, and syndiotactic counterparts have the symmetry axes (as well as the symmetry planes). Polyamides from $\alpha, \omega$-dicarboxylic- $n$ alkanes and $\alpha, \omega$-diamino- $n$-alkanes have the symmetry planes (and the symmetry axes) or the symmetry points, depending on whether the number of carbons in dicarboxylic acids (or in diamines) is odd or even. Thus, for example, polyamide- 66 has the symmetry points. Similar can be said of polyesters and polyethers. Poly(oxyethylene) has all of the three types of symmetry, and this fact rationalizes the results of our numerical computations.

The arguments and conclusions of the preceding two paragraphs remain valid for polymers with deformable side groups, if the symmetries in the planar conformation are preserved by a proper disposition of side groups with respect to the main chain. Examples are stereoregular poly(methyl acrylate), poly(methyl methacrylate), halo-substituted polystyrenes, etc. The symmetries are spoiled for many polymers (for example, the plane symmetry of isotactic vinyl polymers) when groups of the following structure are directly or indirectly connected to the main chain:<smiles>[X]C([X])([X])C</smiles>

where $\mathrm{X}, \mathrm{Y}$, and $\mathrm{Z}$ are different atoms or atomic groups.

We note that polymers with the stated symmetries have no distinguishable direction when viewed along their chain contours. Indeed, for such polymers $\langle\mathbf{r} \cdot \mathbf{u}\rangle_{0}$ should not vary even if the end-to-end vector is measured in the opposite direction, i.e., $\langle\mathbf{r} \cdot \mathbf{u}\rangle_{0}=\langle(-\mathbf{r}) \cdot \mathbf{u}\rangle_{0}$, or hence $\langle\mathbf{r} \cdot \mathbf{u}\rangle_{0}=0$. We conclude therefore that the ex- 
cluded volume has no effect on dipole moments for polymers with no distinguishable direction along chain contours. Stereoirregular vinyl polymers with statistically distributed $d$ and $l$ configurations and with ordinary (not as I above) side groups have no distinguishable direction, and therefore the excluded volume should have no effect on their dipole moments.

Polyamides from $\alpha, \omega$-aminoacids (for example, polyamide-6) and polypeptides are among those polymers which have distinguishable directions. The excluded volume seems to have no serious effect for these polymers for the following reason. Consider first polymers with no deformable side groups. In the trans planar conformation, the resultant dipole moment of each amide group is almost perpendicular to the chain direction and hence $\mathbf{r} \cdot \mathbf{u} \simeq 0$ (see ref 15, pp 185, 189, and 251 ), with the exception of polyproline. Therefore it is also likely that $\langle\mathbf{r} \cdot \mathbf{u}\rangle_{0} \simeq 0$. The situation seems to be little varied by polar side groups connected to skeletal $N$ and/or $C$ inasmuch as $\mathrm{N}-\mathrm{H}$ and $\mathrm{C}-\mathrm{H}$ bonds are almost perpendicular to the chain direction in the planar conformation. To be accurate, however, polyamides and polypeptides require investigation.

It was reported ${ }^{3-7}$ that experimental dipole moments were little affected by the excluded volume for many polymers: poly(vinyl chloride), poly(vinyl acetate), poly(methyl methacrylate), etc., which appear all to have had "atactic" structures. The analysis of this paper explains these experimental results. It also has rationalized and extended Marchal and Benoit's arguments. ${ }^{6}$ It seems rather difficult to find polymers for which $\langle\mathbf{r} \cdot \mathbf{u}\rangle_{0}{ }^{2}\left\langle\boldsymbol{r}^{2}\right\rangle_{0}\left\langle u^{2}\right\rangle_{0}$ departs significantly from zero. If there are, one can test eq 8 , which is plausible but which has not yet been completely justified.

\section{APPENDIX. MULTIVARIATE DISTRIBUTION FUNCTION FOR A REAL POLYMER CHAIN}

A method was developed for the monovariate distribution function. ${ }^{19-21}$ Here we extend it to the case of the multivariate distribution function. Let $\tilde{Z}\left(\mathbf{v}_{1}, \mathbf{v}_{2}, \ldots, \mathbf{v}_{t}\right)$ be the partition function of a polymer chain and $Z\left(\rho_{1}, \rho_{2}, \ldots, \rho_{t}\right)$ be its Fourier transform (except for a constant factor). $Z\left(\rho_{1}, \ldots, \rho_{t}\right)$ can be expressed

$$
\begin{aligned}
& Z\left(\boldsymbol{\rho}_{1}, \ldots, \boldsymbol{\rho}_{t}\right)=\left(8 \pi^{2}\right)^{-1} \\
& \times \iint \exp \left(i \sum_{k=1}^{t} \mathbf{v}_{k} \cdot \boldsymbol{\rho}_{k}\right) \exp (-E / k T) \mathrm{d}\{\theta\} \mathrm{d} \Omega
\end{aligned}
$$

where $E$ is the internal energy of the chain and is a function of a set of internal coordinates $\{\theta\} ; k$ and $T$ are the Boltzman constant and the absolute temperature; $\Omega$ is a set of Eulerian angles which specifies the orientation in space of the chain as a whole; and $i=(-1)^{1 / 2}$.

Expanding $\exp \left(i \sum \mathbf{v}_{k} \cdot \boldsymbol{\rho}_{k}\right)$ in the Taylor series and integrating term-by-term on $\Omega$ and $\{\theta\}$ we find

$$
\begin{aligned}
Z\left(\boldsymbol{\rho}_{1}, \ldots, \boldsymbol{\rho}_{t}\right) & =Z\left[1-\frac{1}{6} \sum_{k, l} q_{k l}\left(\boldsymbol{\rho}_{l} \cdot \boldsymbol{\rho}_{l}\right)+\cdots\right] \\
& =Z \exp \left[-\frac{1}{6} \sum_{k, l} q_{k l}\left(\boldsymbol{\rho}_{k} \cdot \boldsymbol{\rho}_{l}\right)\right]
\end{aligned}
$$

where $Z=Z(0, \ldots, 0)$ is the partition function of the chain in free state and $q_{k l}=\left\langle\mathbf{v}_{l k} \cdot \mathbf{v}_{l}\right\rangle_{0}$, the average referring to that for the free chain. The following relations were used

$$
\begin{gathered}
\int\left(\mathbf{v}_{k} \cdot \boldsymbol{\rho}_{k}\right) \mathrm{d} \Omega=0 \\
\left(8 \pi^{2}\right)^{-1} \iint\left(\mathbf{v}_{k} \cdot \boldsymbol{\rho}_{k}\right)\left(\mathbf{v}_{l} \cdot \boldsymbol{\rho}_{l}\right) \exp (-E / k T) \mathrm{d} \Omega \mathrm{d}\{\theta\} \\
=\frac{1}{3} \int\left(\mathbf{v}_{k} \cdot \mathbf{v}_{l}\right)\left(\boldsymbol{\rho}_{k} \cdot \boldsymbol{\rho}_{l}\right) \exp (-E / k T) \mathrm{d}\{\theta\} \\
=\frac{1}{3} Z q_{k l}\left(\boldsymbol{\rho}_{k} \cdot \boldsymbol{\rho}_{l}\right)
\end{gathered}
$$

The second equality in eq 16 is valid only when, for every $k, \mathbf{v}_{k}$ consists of contributions from many units of the chain and $v_{k}{ }^{2}\left(=\left|\mathbf{v}_{k}\right|^{2}\right)$ is not too large compared with its average value. For further details consult the case previously discussed. ${ }^{19}$

$\tilde{Z}\left(\mathbf{v}_{1}, \ldots, \mathbf{v}_{t}\right)$ is expressed as the inverse Fourier transform of $Z\left(\rho_{1}, \ldots, \rho_{t}\right)$ :

$$
\begin{gathered}
\tilde{Z}\left(\mathbf{v}_{1}, \ldots, \mathbf{v}_{t}\right)=Z\left(8 \pi^{3}\right)^{-t} \int \exp \left[-i \sum_{k=1}^{t} \mathbf{v}_{k} \cdot \boldsymbol{\rho}_{k}\right. \\
\left.\quad-\frac{1}{6} \sum_{k, l} q_{k l}\left(\boldsymbol{\rho}_{k} \cdot \boldsymbol{\rho}_{l}\right)\right] \mathrm{d} \boldsymbol{\rho}_{1} \mathrm{~d} \boldsymbol{\rho}_{2} \cdots \mathrm{d} \boldsymbol{\rho}_{t} \\
=Z\left(8 \pi^{3}\right)^{-t} I_{x} I_{y} I_{z} \\
I_{x}=\int \exp \left(-i \sum_{k=1}^{t} v_{k} \rho_{l}\right. \\
\left.-\frac{1}{6} \sum_{k, l} q_{k l} \rho_{k} \rho_{l}\right) \mathrm{d} \rho_{1} \mathrm{~d} \rho_{2} \cdots \mathrm{d} \rho_{t}
\end{gathered}
$$


In eq 20 we have omitted, for simplicity, the subscript $x$ to be attached to $v_{k}$ and $\rho_{k}$. We can write eq 20 in the vector-matrix form

$$
I_{x}=\int \exp \left(-i \mathbf{v}^{T} \boldsymbol{\rho}-\frac{1}{6} \boldsymbol{\rho}^{T} \mathbf{q} \boldsymbol{\rho}\right) \mathrm{d} \boldsymbol{\rho}
$$

where $\mathbf{v}=\left(\begin{array}{llll}v_{1} & v_{2} \cdots v_{k}\end{array}\right)^{T}, \boldsymbol{\rho}=\left(\begin{array}{llll}\rho_{1} & \rho_{2} & \cdots & \rho_{k}\end{array}\right)^{T}$, and $T$ denotes the transpose. We change variables from $\rho$ to $\sigma$ by an orthogonal transformation $\mathbf{U}$, i.e., $\boldsymbol{\rho}=\mathbf{U} \boldsymbol{\sigma}$ so that $\mathbf{U}^{T} \mathbf{q} \mathbf{U}$ becomes a diagonal matrix, i.e., $\mathbf{U}^{T} \mathbf{q} \mathbf{U}=\lambda=\left\{\lambda_{k i} \delta_{k l}\right\}$. Now,

$$
I_{x}=U \int \exp \left\{-\sum_{k=1}^{t}\left[i\left(\mathbf{v}^{T} \mathbf{U}\right)_{k} \sigma_{k}+\frac{1}{6} \lambda_{k} \sigma_{k}{ }^{2}\right]\right\} \mathrm{d} \boldsymbol{\sigma}
$$

where $\left(\mathbf{v}^{T} \mathbf{U}\right)_{k}$ is the $k$ th element of $\mathbf{v}^{T} \mathbf{U}$ and $U=|\mathbf{U}|$. Upon integration

$$
I_{x}=q^{-1 / 2}(6 \pi)^{t / 2} \exp \left[-\frac{3}{2} \sum_{k=1}^{t}\left(\mathbf{v}^{T} \mathbf{U}\right)_{k}{ }^{2} / \lambda_{k}\right]
$$

Since

$$
\sum_{k=1}^{t}\left(\mathbf{v}^{T} \mathbf{U}\right)_{k}{ }^{2} / \lambda_{k}=\mathbf{v}^{T} \mathbf{U} \lambda^{-1} \mathbf{U}^{T} \mathbf{v}=\mathbf{v}^{T} \mathbf{q}^{-1} \mathbf{v}
$$

we have

$$
\begin{aligned}
I_{x} & =q^{-1 / 2}(6 \pi)^{t / 2} \exp \left(-\frac{3}{2} \mathbf{v}^{T} \mathbf{q}^{-1} \mathbf{v}\right) \\
& =q^{-1 / 2}(6 \pi)^{t / 2} \exp \left[-(3 / 2 q) \sum_{k, l} q^{k l} v_{k x} v_{l x}\right]
\end{aligned}
$$

In eq 24 we have introduced the subscript $x$. Substituting eq 24 and similar expressions for $I_{y}$ and $I_{z}$ into eq 19 , and then dividing by $Z$, we find eq 2 for $P=\tilde{Z}\left(\mathbf{v}_{1}, \ldots, \mathbf{v}_{t}\right) / Z$.

\section{REFERENCES}

1. P. J. Flory, "Principles of Polymer Chemistry," Cornell University Press, Ithaca, New York, N.Y., 1953.
2. M. Kurata and W. H. Stockmayer, Adv. Polym. Sci., 3, 196 (1963).

3. L. de Brouckere, D. Buess, J. de Bock, and J. Versluys, Bull. Soc. Chim. Belges, 64, 669 (1955).

4. L. de Brouckere, D. Buess, and L. K. H. van Beek, J. Polym. Sci., 23, 233 (1957).

5. L. de Brouckere and A. Lecocq-Robert, Bull. Soc. Chim. Belges, 70, 549 (1961).

6. J. Marchal and H. Benoit, J. Chim. Phys., 52, 818 (1955); J. Polym. Sci., 23, 223 (1957).

7. J. Marchal and C. Lapp, J. Polym. Sci., 27, 572 (1958).

8. E. Teramoto, Busseiron Kenkyu (Res. Chem. Phys.), 39, 1 (1951); 40, 18 (1951); 41, 14 (1951); 44, 36 (1951).

9. M. Fixman, J. Chem. Phys., 23, 1656 (1955).

10. T. Oku, Busseiron Kenkyu (Res. Chem. Phys.), Series 2, 7, 260(1960).

11. K. Okano and Y. Tanabe, Preprints, SPSJ 16th Symposium on Macromolecules, Japan, Fukuoka, 1967, Part II, p 440.

12. H. Yamakawa and G. Tanaka, J. Chem. Phys., 47, 3991 (1967).

13. K. Nagai, J. Phys. Chem., 74, 3411 (1970).

14. K. Nagai, J. Chem. Phys., 51, 1091 (1969).

15. P. J. Flory, "Statistical Mechanics of Chain Molecules," Interscience, New York, N.Y., 1969.

16. W. H. Stockmayer, Pure Appl. Chem., 15, 539 (1967); Ref 2, p 199.

17. J. E. Mark and P. J. Flory, J. Amer. Chem. Soc., 87, 1415 (1965).

18. J. E. Mark and P. J. Flory, J. Amer. Chem. Soc., 88, 3702 (1966).

19. K. Nagai, J. Chem. Phys., 38, 924 (1963).

20. K. Nagai, J. Chem. Phys., 40, 2818 (1964).

21. Ref 15 , Chap. VIII. 